\title{
Self medication practices and its determinants in health care professionals during the coronavirus disease-2019 pandemic: cross-sectional study
}

\author{
Ogochukwu Chinedum Okoye ${ }^{1}$. Oluseyi Ademola Adejumo ${ }^{2}$ (D) Abimbola Olubukunola Opadeyi ${ }^{3}$. \\ Cynthia Roli Madubuko ${ }^{4}$ - Maureen $\mathrm{Ntaji}^{5}$ (1) Kenechukwu Chukwuemeka Okonkwo ${ }^{2}$.

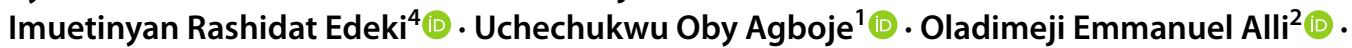 \\ John Oghenevwirhe Ohaju-Obodo ${ }^{1}$ [D
}

Received: 16 September 2021 / Accepted: 23 December 2021 / Published online: 12 January 2022

(c) The Author(s), under exclusive licence to Springer Nature Switzerland AG 2022

\begin{abstract}
Background The exposure of health care professionals (HCP) to patients with coronavirus disease-2019 (COVID-19) in the course of performing their professional duties may expose them to contracting the virus. This may likely increase their tendency to self-medicate for prevention or treatment of perceived infection. Aim This study determined the prevalence of COVID-19 related self-medication and its determinants among HCPs in three tertiary hospitals in Southern Nigeria. Method This was a cross-sectional study that enrolled 669 adult HCPs from three tertiary hospitals in three Southern Nigerian States using a non-probability convenience sampling method. A structured self-administered questionnaire was used for data collection. Data entry and analysis were done using IBM SPSS version 22. Results The mean age of the respondents was $35.6 \pm 8.7$ years. Two hundred and forty-three respondents (36.3\%) reported having practiced COVID-19 related self-medication. The commonly used medications were ivermectin, azithromycin, vitamin $\mathrm{C}$, chloroquine and zinc. Factors associated with self-medication were older age $(p=<0.0001)$, being pharmacist $(p=0.03)$, higher income $(p=<0.0001)$, previous COVID-19 testing $(p<0.001)$. Predictors of self medication were $>44$ years (Adjusted Odd Ratio[AOR]:2.77,95\% Confidence Interval [CI]: 1.62-4.75, $p=<0.0001$ ), previous COVID-19 testing (AOR =2.68, 95\% CI: $1.82-3.94, p=<0.0001$ ). Conclusion About one-third of HCPs practiced COVID-19 related self-medication. HCPs that are often assumed to be health literate may not necessarily practice safe health behavior. Regular health education of the HCPs on implications of self-medications is highly recommended. There should also be formulation and effective implementation of policies that regulate purchase of medications.
\end{abstract}

Keywords COVID-19 $\cdot$ Health care professionals $\cdot$ Nigeria $\cdot$ Self-medication

\section{Impacts on practice}

Oluseyi Ademola Adejumo

oluseyiadejumo2017@gmail.com

1 Department of Internal Medicine, Delta State University Teaching Hospital Oghara, Oghara, Delta State, Nigeria

2 Department of Internal Medicine, University of Medical Sciences Teaching Hospital, Ondo, Ondo State, Nigeria

3 Department of Clinical Pharmacology and Therapeutics, University of Benin, Benin City, Edo State, Nigeria

4 Department of Internal Medicine, University of Benin Teaching Hospital, Benin City, Edo State, Nigeria

5 Department of Community Medicine, Delta State University Teaching Hospital Oghara, Oghara, Delta State, Nigeria
- Health education on adverse implications of self-medications should focus on both the HCPs and the general population.

- There should be formulation and/or implementation of policies that will ensure the regulation of purchase of medications in Nigeria. 


\section{Introduction}

The exposure of health care professionals (HCP) to patients with coronavirus disease-2019 (COVID-19) in the course of performing their professional duties may expose them to contracting the virus. This may likely increase their tendency to self-medicate for prevention or treatment of perceived infection [1]. This is in spite of the non-pharmacological and risk stratification measures in place by some institutions [2]. Self-medication is the use of medicinal products, including over the counter (OTC) or non-prescription medicines as well as prescription only medicines (POM) to treat selfdiagnosed symptoms or illnesses without obtaining advice from a physician, pharmacist or other HCPs in the diagnosis or treatment. It also includes the continuous or intermittent use of a medication previously prescribed by a physician for chronic or recurring symptoms or diseases [3].

There are benefits associated with self-medication such as the active choice of patients' utilization of the products, direct and rapid access to treatment and self-care, convenience and reduction in costs associated with hospital visitations [3]. Self-medication is associated with risk that may predispose to increased morbidity and mortality from wrong diagnosis, delay in seeking appropriate medical advice and treatment [4]. There is also a risk of antimicrobial resistance and adverse drug reactions, higher probability of drugdrug interactions, increased medication errors and medical expenditure [4].

The practice of self-medication is common among Nigerian consumers and HCPs [5-10]. Consumers of medicines in Nigeria access care and purchase medicines from various sources such as patent medicine stores (PMS), pharmacies, public and private hospitals. However, majority would rather patronize PMS and community pharmacies than health care facilities due to poor access to public health facilities, long waiting time, unavailability of essential medicines at hospitals and the high cost associated with visiting private health facilities [11, 12]. Poor and ineffective medicine regulatory system allows the availability and purchase of POM in PMS and community pharmacies without a prescription [13].

HCPs are expected to educate the populace on the dangers of self-medication. However, HCPs have medical needs just like their clients and have personal preferences for treatment. These preferences may be informed by quest for personal satisfaction as against evidence-based knowledge. The nature of their training empowers them to recognize and hopefully seek treatment earlier than the uninformed individual. While this may be beneficial in most instances, it may also adversely affect their health as HCPs have poor health-seeking abilities [14].

During the first wave of SARS-COV2 pandemic, HCPs were globally reported to have a high risk of contracting the infection with attendant morbidity and mortality which were worse in HCPs with pre-morbid conditions [15]. The ensuing anxiety associated with the fear of contracting SARSCOV2 was aggravated by conflicting literature on natural course of the disease, prevention, as well as treatment strategies for the SARS-COV2 virus [16]. Several clinical trials which were not conclusive further worsened the anxiety regarding the treatment of the disease $[17,18]$.

The Nigerian Centre for Disease Control (NCDC) guidelines advised symptomatic relief of fever, cough and nasal congestion with antipyretics. Also, cough medicines, multivitamins and mineral supplements were recommended in mild cases [19]. The use of empiric antibiotic therapy and conservative fluid management was recommended for treatment of severe cases. The NCDC however, strongly advised against the use of chloroquine, hydroxychloroquine, azithromycin, antivirals such as lopinavir/ritonavir, immunomodulators, plasma therapy except in the setting of a clinical trial for treatment of COVID-19 because the efficacy of these medications were not proven.

Some previous studies showed that self-medication was more prevalent during the COVID-19 pandemic $[1,13,20]$. A study of community pharmacies in Edo state, Nigeria showed an increase in the purchase of some medications considered useful for COVID-19 treatment or prophylaxis in the COVID-19 era compared to pre-COVID-19 period [21]. It is unclear if the HCPs in Nigeria practiced self medication to treat COVID-19 symptoms during the pandemic as few studies have evaluated self-medication use by HCPs in the COVID-19 pandemic.

\section{Aim}

This study determined the prevalence of COVID-19 related self-medication and its determinants among HCPs in three tertiary hospitals in Southern Nigeria.

\section{Ethics approval}

The Research and Ethics Committee of University of Benin Teaching Hospital, Benin City, Edo State, Nigeria and Delta State University Abraka, Delta State, Nigeria approved the questionnaire and methodology of this study. The ethical protocol numbers are ADM/E22/A/VOLVII/14831123 and REC/FBMS/DELSU/21/101. Written informed consent was obtained from each participant after explanation of the purpose and procedure of the study and before data collection. All data collection tools were anonymous, restricted within the research team only and stored safely to ensure confidentiality. 


\section{Method}

\section{Study area}

This study was carried out between March and April 2021 in three tertiary hospitals located in Southern Nigeria namely; Delta State University Teaching Hospital Oghara (DELSUTH), University of Benin Teaching Hospital Benin (UBTH), and University of Medical Sciences Teaching Hospital Complex, Ondo (UNIMEDTHC). These hospitals are teaching hospitals that offer specialized and tertiary medical services.

\section{Study design and participants}

This study was cross-sectional and descriptive. Cochran formula for descriptive studies was used in calculating the minimum sample size. The minimum sample size was 422 after inclusion of an assumed non-response rate of $10 \%$. The low non-response rate was adopted because the study population was HCPs which were expected to be easily accessible and more co-operative compared to a general population. Eight hundred questionnaires (approximately twice the minimum sample size) were shared among the three centres in the ratio 1.5:1.5:1 based on their respective staff-strength. The number 800 was chosen for ease of allocation.

The study participants were HCPs which included doctors, nurses, pharmacists, laboratory scientists, and health assistants who were employed by the three hospitals and gave consent to participate in the study. A non-probability convenience sampling method was used to enroll participants with the help of trained research assistants.

\section{Data collection instrument}

Data was collected using a researcher-developed semi-structured questionnaire which was piloted among HCPs who did not participate in the study. The questionnaires were selfadministered and data collected included socio-demographic data, behavioural history (alcohol and smoking), past medical history including hypertension, diabetes mellitus, and any other existing chronic medical illness (open ended question). COVID-19 related questions included knowledge of COVID-19, history of COVID-19 testing, details of self-medication related to COVID-19 done during the period of the pandemic and history of self-medication that was unrelated to COVID-19. The primary outcome was self-medication for COVID-19. This was defined according to the WHO guidelines [3]. The diagnosis of hypertension, diabetes mellitus and other co-morbidities were based on self-report of participants.

\section{Data analysis}

Data were analysed using the SPSS 22.0 (IBM SPSS Statistics, New York, USA). Data from all returned questionnaires $(n=669)$ were entered into Excel spreadsheet and exported to SPSS 22.0. Missing data for individual variables occurred randomly and were automatically excluded during data analysis by the statistical software. Descriptive statistics and inferential statistics were used as appropriate to analyse the data. The occurrence of self-medication for COVID19 amongst respondents was presented as frequencies and percentages according to socio-demographic and clinical characteristics. The Chi-square test was used to determine the relationship between participants' characteristics and the practice of self-medication for COVID-19. The logistic regression model was used to determine independent risk factors of self-medication.

\section{Results}

\section{Characteristics of participants}

A total of 669 out of 800 questionnaires were returned giving a response rate of $83.6 \%$. Three hundred and seventy-four out of 638 respondents who provided information on sex were females (58.6\%). Overall mean age was $35.6 \pm 8.7$ years and most respondents $(42.4 \%)$ were between 30-39 year age group. Over half of the respondents (58.1\%) were married and $97.5 \%$ had attained tertiary level education. Doctors (55.9\%) and nurses (37.0\%) were in the majority, while records clerks $(0.02 \%)$ and pharmacists $(0.04 \%)$ were in the minority. Table 1 summarises the socio-demographic characteristics of respondents. Sixty-four (9.6\%) and $1.6 \%$ of respondents reported they were hypertensive and diabetic respectively.

\section{Self-medication for COVID-19}

Two hundred and forty-three respondents (36.3\%) reported taking medicines for COVID-19 prevention and/or treatment; three-quarters of the respondents did not supply name of medication used (Table 2). Medicines used included ivermectin (9.5\%), azithromycin (9.1\%), vitamin C (7.4\%), chloroquine $(5.7 \%)$, and zinc $(2.0 \%)$. The medicines used were obtained from pharmacies (80.8\%), patent medicine shops (17\%) and friends/family (2.2\%). The reasons given for taking the medicines included for prophylaxis (45.6\%), definite exposure $(31.2 \%)$, symptoms $(21.3 \%)$, and probable exposure (15.2\%) amongst others (Table 2). Majority (84.7\%) reported that medicines taken were beneficial. 
Table 1 Demographic and characteristics of respondents

\begin{tabular}{|c|c|c|c|c|c|c|c|c|}
\hline \multirow[t]{2}{*}{ Centres } & \multicolumn{2}{|l|}{ A } & \multicolumn{2}{|l|}{ B } & \multicolumn{2}{|l|}{$\underline{\mathrm{C}}$} & \multicolumn{2}{|l|}{$\underline{\text { Total }}$} \\
\hline & $n$ & $\%$ & $n$ & $\%$ & $n$ & $\%$ & $n$ & $\%$ \\
\hline \multicolumn{9}{|l|}{ Gender } \\
\hline Male & 97 & 41.3 & 127 & 41.9 & 40 & 40.0 & 264 & 41.4 \\
\hline Female & 138 & 58.7 & 176 & 58.1 & 60 & 60.0 & 374 & 58.6 \\
\hline Total & 235 & 100.0 & 303 & 100.0 & 100 & 100.0 & 638 & 100.0 \\
\hline \multicolumn{9}{|l|}{ Age (years) } \\
\hline $20-29$ & 66 & 29.1 & 76 & 25.4 & 27 & 39.1 & 169 & 28.4 \\
\hline $30-39$ & 88 & 38.8 & 134 & 44.8 & 30 & 43.5 & 252 & 42.4 \\
\hline $40-49$ & 42 & 18.5 & 67 & 22.4 & 9 & 13.0 & 118 & 19.8 \\
\hline $50-59$ & 30 & 13.2 & 21 & 7.0 & 3 & 4.3 & 54 & 9.1 \\
\hline$\geq 60$ & 1 & 0.4 & 1 & 0.3 & 0 & 0.0 & 2 & 0.3 \\
\hline Total & 226 & 100.0 & 299 & 100.0 & 69 & 100.0 & 595 & 100.0 \\
\hline Mean \pm SD & $36.3 \pm 9.5$ & & $35.6 \pm 8.4$ & & $33.0 \pm 7.4$ & & $35.6 \pm 8.7$ & \\
\hline \multicolumn{9}{|l|}{ Marital status } \\
\hline Never married & 92 & 39.5 & 100 & 33.0 & 61 & 46.9 & 253 & 38.0 \\
\hline Currently married & 128 & 54.9 & 192 & 63.4 & 67 & 51.5 & 387 & 58.1 \\
\hline Domestic partner & 1 & 0.4 & 1 & 0.3 & 2 & 1.5 & 4 & 0.6 \\
\hline Separated & 3 & 1.3 & 4 & 1.3 & 0 & 0.0 & 7 & 11 \\
\hline Divorced & 0 & 0.0 & 2 & 0.7 & 0 & 0.0 & 2 & 0.3 \\
\hline Widowed & 9 & 3.9 & 4 & 1.3 & 0 & 00 & 13 & 2.0 \\
\hline Total & 233 & 100.0 & 303 & 100.0 & 130 & 100.0 & 666 & 100.0 \\
\hline \multicolumn{9}{|l|}{ Educational status } \\
\hline None & 0 & 0.0 & 0 & 0.0 & 1 & 0.8 & 1 & 0.1 \\
\hline Primary & 6 & 2.6 & 0 & 0.0 & 0 & 0.0 & 2 & 2.6 \\
\hline Secondary & 4 & 1.7 & 6 & 2.0 & 0 & 0.0 & 10 & 1.5 \\
\hline Tertiary & 225 & 95.7 & 297 & 98.0 & 129 & 99.2 & 651 & 97.5 \\
\hline Total & 235 & 100.0 & 303 & 100.0 & 130 & 100.0 & 664 & 100.0 \\
\hline \multicolumn{9}{|l|}{ Occupation } \\
\hline Doctor & 104 & 44.3 & 113 & 37.3 & 71 & 55.9 & 288 & 55.9 \\
\hline Nurse & 61 & 26.0 & 121 & 39.9 & 47 & 37.0 & 229 & 37.0 \\
\hline Pharm & 17 & 7.2 & 9 & 3.0 & 0 & 0.0 & 26 & 0.0 \\
\hline MLS & 8 & 3.4 & 14 & 4.6 & 2 & 1.6 & 24 & 1.6 \\
\hline Health assistant & 12 & 5.1 & 9 & 3.0 & 6 & 4.7 & 27 & 4.7 \\
\hline Records officers & 0 & 0.0 & 13 & 4.3 & 0 & 0.0 & 13 & 0.0 \\
\hline Others** & 33 & 14.0 & 24 & 7.9 & 1 & 0.8 & 58 & 0.8 \\
\hline Total & 235 & 100.0 & 303 & 100.0 & 127 & 100.0 & 665 & 100.0 \\
\hline \multicolumn{9}{|l|}{ Income } \\
\hline$<600,000$ & 23 & 10.0 & 9 & 3.0 & 16 & 14.4 & 48 & 7.4 \\
\hline $600,000-1.19 \mathrm{M}$ & 55 & 23.8 & 67 & 22.1 & 25 & 22.5 & 147 & 22.8 \\
\hline $1.20-2.39 \mathrm{M}$ & 94 & 40.7 & 101 & 33.3 & 38 & 34.2 & 233 & 36.1 \\
\hline $2.40-5.99 \mathrm{M}$ & 43 & 18.6 & 75 & 24.8 & 29 & 26.1 & 147 & 22.8 \\
\hline $6-12 \mathrm{M}$ & 16 & 6.9 & 47 & 15.5 & 1 & 0.9 & 64 & 9.9 \\
\hline$>12 \mathrm{M}$ & 0 & 0.0 & 4 & 1.3 & 2 & 1.8 & 6 & 0.9 \\
\hline Total & 231 & 100.0 & 303 & 100.0 & 111 & 100.0 & 645 & 100.0 \\
\hline
\end{tabular}

$\mathrm{M}=$ Million $\mid \mathrm{MLS}^{\ddagger}=$ Medical Laboratory Scientist I Pharm $=$ Pharmacist |Others** = Dietician, Engineer, Dentist, Medical Laboratory Technician, Pharm Tech, Physiotherapist, Psychologist, Social worker 
Table 2 Self-medication for COVID-19 among the HCPs

\begin{tabular}{|c|c|c|}
\hline Parameter & Frequency $(n)$ & Percentage $(\%)$ \\
\hline \multicolumn{3}{|l|}{ Self-medication } \\
\hline Yes & 243 & 36.3 \\
\hline No & 423 & 63.2 \\
\hline No response & 3 & 0.5 \\
\hline Total & 669 & 100.0 \\
\hline \multicolumn{3}{|l|}{ Names of medications used } \\
\hline Zinc Sulphate & 5 & 2.0 \\
\hline Vitamin C & 18 & 7.4 \\
\hline Chloroquine & 14 & 5.7 \\
\hline Azithromycin & 22 & 9.1 \\
\hline Ivermectin & 23 & 9.5 \\
\hline No response & 161 & 66.2 \\
\hline Total & 243 & 100.0 \\
\hline \multicolumn{3}{|l|}{ Reason for taking medication* } \\
\hline Symptoms of COVID & 52 & 21.3 \\
\hline Definite exposure to a patient diagnosed with COVID & 76 & 31.2 \\
\hline Probable exposure to a patientdiagnosed with COVID & 37 & 15.2 \\
\hline Prophylaxis against contracting COVID-19 & 111 & 45.6 \\
\hline Psychological assurance & 17 & 6.9 \\
\hline No reason & 9 & 3.7 \\
\hline \multicolumn{3}{|l|}{ Was taking the medication beneficial? } \\
\hline Yes & 199 & 81.9 \\
\hline No & 31 & 12.8 \\
\hline No response & 13 & 5.3 \\
\hline Total & 243 & 100.0 \\
\hline
\end{tabular}

*Multiple responses

\section{Factors associated with self-medication for COVID-19}

A higher proportion of respondents in older age groups practiced self-medication compared to younger age-groups ( $p=<0.0001$ ); $30.9 \%$ and $33.4 \%$ of those in the $20-29$ years and 30-39 years age-group respectively, practiced self-medication compared to $54.1 \%, 50 \%$ and $100 \%$ among those is $40-49,50-59$ and $>60$ years age-group respectively (Table 3).

Self-medication was lowest among HCP who were never married $(28.6 \%)$ and the widows $(38.4 \%)$, compared to those currently married (41.4\%), divorced $(50 \%)$ or separated $(71 \%)$. Self-medication practice varied among the categories of health workers; it was highest among pharmacists (50\%) and lowest among health assistants (14.8\%), $P=0.03$. Self-medication was lowest (17.8\%) among those earning the lowest annual income $(<\mathrm{N} 600,000)$ compared to those with higher annual income -N600,000-1,199,999 $(34.9 \%), \quad \mathrm{N} 1,200,000-2,399,999 \quad(34.2 \%)$, and N6,000,000-11,999,999(65.6\%), Table 3.
Self-medication was more common among respondents who had COVID-19 test (45.7\%) compared to those who had not $(29.7 \%), P=<0.0001$. Similarly, self-medication was significantly commoner among hypertensive HCP (51.5\%) compared to non-hypertensive HCP $(35.4 \%) p=0.039$; and diabetic HCP $(81.8 \%)$ compared to non-diabetic HCP (35.9\%) $p=0.004$ (Table 4).

\section{Independent risk factors of COVID-19 Self-medication}

HCP who were $>44$ years had greater odds of practicing self-medication compared to younger ones $(\mathrm{AOR}=2.77$, 95\% CI: $1.62-4.75, P=<0.0001)$. HCP who had tested for COVID-19 had greater odds of practising self-medication compared to those who had not tested $(\mathrm{OR}=2.68,95 \%$ CI: $1.82-3.94, P=<0.0001)$. Those with the least annual income had lesser odds of practicing self-medication compared to those who earned more, but this was not statistically 
Table 3 Socio-demographic factors affecting self medication for COVID-19

\begin{tabular}{|c|c|c|c|c|c|c|c|c|}
\hline & \multicolumn{2}{|c|}{ Self-medication } & \multicolumn{2}{|c|}{$\begin{array}{l}\text { No Self-medi- } \\
\text { cation }\end{array}$} & \multicolumn{2}{|c|}{ Total } & \multirow[t]{2}{*}{ Test statistics } & \multirow[t]{2}{*}{$P$-value } \\
\hline & $n$ & $\%$ & $n$ & $\%$ & $n$ & $\%$ & & \\
\hline \multicolumn{9}{|l|}{ Gender } \\
\hline Male & 94 & 40.0 & 167 & 42.3 & 261 & 41.4 & 0.315 & 0.574 \\
\hline Female & 141 & 60.0 & 228 & 57.7 & 369 & 58.6 & & \\
\hline TOTAL & 235 & 100.0 & 395 & 100.0 & 630 & 100.0 & & \\
\hline \multicolumn{9}{|l|}{ Age (years) } \\
\hline $20-29$ & 52 & 22.7 & 116 & 32.0 & 168 & 28.4 & 25.855 & $<0.0001$ \\
\hline $30-39$ & 84 & 36.7 & 167 & 46.0 & 251 & 42.4 & & \\
\hline $40-49$ & 64 & 27.9 & 53 & 14.6 & 117 & 19.8 & & \\
\hline $50-59$ & 27 & 11.8 & 27 & 7.4 & 54 & 9.1 & & \\
\hline \multirow[t]{2}{*}{$\geq 60$} & 2 & 0.9 & 0 & 0.0 & 2 & 0.3 & & \\
\hline & 229 & 100.0 & 363 & 100.0 & 592 & 100.0 & & \\
\hline \multicolumn{9}{|l|}{ Marital status } \\
\hline Never married & 70 & 28.9 & 175 & 42.4 & 245 & 37.4 & 14.680 & 0.012 \\
\hline Currently married & 159 & 65.7 & 225 & 54.5 & 384 & 58.6 & & \\
\hline Domestic partner & 2 & 0.8 & 2 & 0.5 & 4 & 0.6 & & \\
\hline Separated & 5 & 2.1 & 2 & 0.5 & 7 & 1.1 & & \\
\hline Divorced & 1 & 0.4 & 1 & 0.2 & 2 & 0.3 & & \\
\hline \multirow[t]{2}{*}{ Widowed } & 5 & 2.1 & 8 & 1.9 & 13 & 2.0 & & \\
\hline & 242 & 100.0 & 413 & 100.0 & 655 & 100.0 & & \\
\hline \multicolumn{9}{|l|}{ Educational status } \\
\hline None & 0 & 0.0 & 1 & 0.2 & 1 & 0.2 & 4.849 & 0.183 \\
\hline Primary & 1 & 0.4 & 5 & 1.2 & 6 & 0.9 & & \\
\hline Secondary & 1 & 0.4 & 9 & 2.2 & 10 & 1.5 & & \\
\hline \multirow[t]{2}{*}{ Tertiary } & 240 & 99.2 & 400 & 96.4 & 640 & 97.4 & & \\
\hline & 242 & 100.0 & 415 & 100.0 & 657 & 100.0 & & \\
\hline \multicolumn{9}{|l|}{ Occupation } \\
\hline Doctor & 94 & 38.7 & 185 & 44.9 & 279 & 42.7 & 32.585 & 0.037 \\
\hline Nurse & 96 & 39.5 & 131 & 31.8 & 227 & 34.7 & & \\
\hline Pharmacist & 13 & 5.4 & 13 & 3.2 & 26 & 4.0 & & \\
\hline $\mathrm{MLS}^{\ddagger}$ & 7 & 2.9 & 17 & 4.1 & 24 & 3.7 & & \\
\hline Health assistant & 4 & 1.6 & 23 & 5.7 & 27 & 4.1 & & \\
\hline Record officers & 4 & 1.6 & 9 & 2.2 & 13 & 2.0 & & \\
\hline \multirow[t]{2}{*}{ Others** } & 25 & 10.3 & 33 & 8.1 & 58 & 8.8 & & \\
\hline & 243 & 100.0 & 411 & 100.0 & 654 & 100.0 & & \\
\hline \multicolumn{9}{|l|}{ House hold size } \\
\hline $0-3$ & 106 & 44.0 & 158 & 40.0 & 264 & 41.5 & 1.573 & 0.455 \\
\hline $4-6$ & 120 & 49.8 & 204 & 51.6 & 324 & 50.9 & & \\
\hline \multirow[t]{2}{*}{7 or more } & 15 & 6.2 & 33 & 8.4 & 48 & 7.5 & & \\
\hline & 241 & 100.0 & 395 & 100.0 & 636 & 100.0 & & \\
\hline \multicolumn{9}{|l|}{ Income } \\
\hline$<600,000$ & 8 & 3.4 & 37 & 9.3 & 45 & 7.1 & 30.691 & $<0.0001$ \\
\hline $600,000-1.19 \mathrm{M}$ & 51 & 21.7 & 95 & 23.8 & 146 & 23.0 & & \\
\hline $1.2 \mathrm{M}-2.39 \mathrm{M}$ & 78 & 33.2 & 150 & 37.5 & 228 & 35.9 & & \\
\hline $2.4 \mathrm{M}-5.99 \mathrm{M}$ & 54 & 23.0 & 92 & 23.0 & 146 & 23.0 & & \\
\hline $6.0 \mathrm{M}-11.9 \mathrm{M}$ & 42 & 17.9 & 22 & 5.5 & 64 & 10.1 & & \\
\hline \multirow[t]{2}{*}{$>12 \mathrm{M}$} & 2 & 0.9 & 4 & 1.0 & 6 & 0.9 & & \\
\hline & 235 & 100.0 & 400 & 100.0 & 635 & 100.0 & & \\
\hline
\end{tabular}

$\chi^{2}=$ Pearson Chi-Square test. $\mid \mathrm{t}=$ Student's T-test $\mid P$-value $=$ Probability value $\mid P=$ Probability value of statistical significance | $\mathrm{M}=$ Million $\mid \mathrm{MLS}^{\ddagger}=$ Medical Laboratory Scientist | Others** = Dietician, Engineer, Dentist, Medical Laboratory Technician, Pharm Tech, Physiotherapist, Psychologist, Social worker 
Table 4 Behavioural and clinical characteristics and COVID-19 self medication

\begin{tabular}{|c|c|c|c|c|c|c|c|c|}
\hline & \multicolumn{2}{|c|}{ Self-medication } & \multicolumn{2}{|c|}{ No Self-medication } & \multicolumn{2}{|c|}{ TOTAL } & \multirow[t]{2}{*}{$\chi^{2}$} & \multirow[t]{2}{*}{$P$-value } \\
\hline & $n$ & $\%$ & $n$ & $\%$ & $n$ & $\%$ & & \\
\hline \multicolumn{9}{|c|}{ Knowledge of the existence of COVID-19 } \\
\hline Yes & 239 & 98.8 & 405 & 98.1 & 644 & 98.3 & 0.449 & 0.503 \\
\hline No & 3 & 1.2 & 8 & 1.9 & 11 & 1.7 & & \\
\hline Total & 242 & 100.0 & 413 & 100.0 & 655 & 100.0 & & \\
\hline \multicolumn{9}{|c|}{ Testing for COVID-19 } \\
\hline Yes & 135 & 55.6 & 160 & 38.6 & 295 & 44.8 & 17.911 & $<0.0001$ \\
\hline No & 108 & 44.4 & 255 & 61.4 & 363 & 55.2 & & \\
\hline Total & 243 & 100.0 & 415 & 100.0 & 658 & 100.0 & & \\
\hline \multicolumn{9}{|c|}{ History of self-medication } \\
\hline Yes & 92 & 38.2 & 139 & 36.7 & 231 & 37.3 & 0.142 & 0.707 \\
\hline No & 149 & 61.8 & 240 & 63.3 & 389 & 62.7 & & \\
\hline Total & 241 & 100.0 & 379 & 100.0 & 620 & 100.0 & & \\
\hline \multicolumn{9}{|c|}{ Hypertension } \\
\hline Yes & 33 & 13.7 & 31 & 7.5 & 64 & 9.8 & 6.510 & $* 0.039$ \\
\hline No & 208 & 86.3 & 380 & 92.5 & 588 & 90.2 & & \\
\hline Total & 241 & 100.0 & 411 & 100.0 & 652 & 100.0 & & \\
\hline \multicolumn{9}{|c|}{ Diabetes mellitus } \\
\hline Yes & 9 & 3.8 & 2 & 0.5 & 11 & 1.7 & 10.934 & $* 0.004$ \\
\hline No & 230 & 96.2 & 410 & 99.5 & 640 & 98.3 & & \\
\hline Total & 239 & 100.0 & 412 & 100.0 & 651 & 100.0 & & \\
\hline
\end{tabular}

$\chi^{2}=$ Pearson Chi-Square test. $\mid P$-value $=$ Probability value $\mid$ significant $(\mathrm{OR}=0.41,95 \% \mathrm{CI}: 0.16-1.01, P=0.054)$ (Table 5).

\section{Discussion}

Our study assessed self-medication practices and determinants among HCPs during the COVID-19 period in three tertiary hospitals in Southern Nigeria. Self-medication for COVID-19 prevention or treatment was practiced by $36.3 \%$ of HCPs in this study. This is similar to $36.2 \%$ reported among the general population in Kenya by Onchonga et al. [1]. However, this is lower than $51.9 \%$ reported by Sadio et al. [22] among HCPs in Togo. Self-medication among HCPs in this study is also lower than the reported prevalence of $41 \%$ among general population in Nigeria [10]. The differences in the prevalence of COVID-19 treatment and prevention related self-medication in these studies may be related to differences in the study participants; knowledge, perception and beliefs about COVID-19; accessibility to medications without prescription; and the presence and enforcement of regulations on drug procurement in different countries.

It is important to note that about half of the HCPs in our study practiced COVID-19 related self-medication as prophylaxis despite the fact that no drug was recommended by the World Health Organization (WHO). Inappropriate use of medications for prophylaxis has potential adverse implications [4]. The possible factors that could have influenced high level of self medication for COVID-19 among HCPs in this study include uncertainties about the novel infection, high level of anxiety among the general population, high rate of infection among HCPs, especially the frontline workers and misinformation by the social media [16, 23-25].

A higher proportion of those who used COVID-19 related self-medication claimed it was beneficial to them. This is similar to report by Makowska et al. [26] in a study done among general population in Poland. However, the perceived benefit among our respondents may be psychological because only about a third of those that used self-medication had definite exposure to COVID-19 while the others who assumed they had COVID-19 exposure may have a different diagnosis.

The drugs commonly used by our study participants to prevent or treat COVID-19 were ivermectin, azithromycin, vitamin $\mathrm{C}$, chloroquine and zinc. This observation corroborated the report of Osaigbovo et al. [21] which showed that there was increase in sales of anti-malarial, antibiotics and multivitamins in some community pharmacies in Benin City, Edo State, Nigeria in the second quarter of year 2020 compared to the first quarter. This second quarter of year 2020 coincided with the period after WHO declared COVID-19 as a pandemic in March 2020 [27].This pattern of self-medication is largely similar to that of previous 
Table 5 Independent risk factors of COVID-19 selfmedication

\begin{tabular}{|c|c|c|c|c|c|c|}
\hline \multirow[t]{2}{*}{ Parameters } & \multirow[t]{2}{*}{ B } & \multirow[t]{2}{*}{ Std. error } & \multirow[t]{2}{*}{$P$-value } & \multirow[t]{2}{*}{ AOR } & \multicolumn{2}{|c|}{$95 \%$ C.I } \\
\hline & & & & & Lower & Upper \\
\hline \multicolumn{7}{|l|}{$\operatorname{Sex}$} \\
\hline Male & -0.193 & 0.217 & 0.373 & 0.82 & 0.54 & 1.26 \\
\hline \multicolumn{7}{|c|}{ Female (Ref) } \\
\hline \multicolumn{7}{|c|}{ Age group (years) } \\
\hline$>44$ & 1.019 & 0.275 & $<0.0001^{*}$ & 2.77 & 1.62 & 4.75 \\
\hline \multicolumn{7}{|l|}{$\leq 44(\operatorname{Ref})$} \\
\hline \multicolumn{7}{|l|}{ Married } \\
\hline Not married & -0.302 & 0.206 & 0.143 & 0.74 & 0.49 & 1.11 \\
\hline \multicolumn{7}{|c|}{ Currently married (Ref) } \\
\hline \multicolumn{7}{|l|}{ Occupation } \\
\hline Doctor & -0.225 & 0.308 & 0.464 & 0.79 & 0.44 & 1.46 \\
\hline Nurse & -0.040 & 0.320 & 0.900 & 0.96 & 0.51 & 1.80 \\
\hline Pharmacist & 0.282 & 0.509 & 0.579 & 1.33 & 0.49 & 3.59 \\
\hline $\operatorname{MLS}^{\ddagger}$ & -0.411 & 0.560 & 0.463 & 0.66 & 0.22 & 1.99 \\
\hline \multicolumn{7}{|c|}{ Others** (Ref) } \\
\hline \multicolumn{7}{|c|}{ Annual income } \\
\hline$<600,000$ & -0.898 & 0.466 & 0.054 & 0.41 & 0.16 & 1.01 \\
\hline \multicolumn{7}{|c|}{$>600,000$ (Ref) } \\
\hline \multicolumn{7}{|c|}{ Knowledge on COVID } \\
\hline Yes & 0.453 & 0.682 & 0.506 & 1.57 & 0.41 & 5.99 \\
\hline \multicolumn{7}{|l|}{ No (Ref) } \\
\hline \multicolumn{7}{|c|}{ Testing for COVID-19 } \\
\hline Yes & 0.988 & 0.196 & $<0.0001^{*}$ & 2.68 & 1.82 & 3.94 \\
\hline \multicolumn{7}{|l|}{ No (Ref) } \\
\hline \multicolumn{7}{|c|}{ History of hypertension } \\
\hline Yes & -0.428 & 1.041 & 0.681 & 0.65 & 0.08 & 5.01 \\
\hline \multicolumn{7}{|l|}{ No (Ref) } \\
\hline \multicolumn{7}{|c|}{ History of diabetes } \\
\hline Yes & 0.073 & 1.521 & 0.962 & 1.07 & 0.05 & 21.18 \\
\hline No (Ref) & & & & & & \\
\hline
\end{tabular}

$\mathrm{B}=$ Unstandardized beta $(\mathrm{B}) \mid \mathrm{SE}=$ Standard Error $\mid P$-value $=$ Probability value $\mid *=$ Probability value of statistical significance $\mid \mathrm{OR}=$ adjusted Odd Ratio studies from both Nigeria and Togo, but there were few differences $[10,22]$. For example, ivermectin was not part of the commonly used medication in the studies by Wegbon et al. [10] and Sadio et al. [22] which were conducted among the general population in Nigeria and Togo, respectively. This may be due to the fact that different therapies are being developed and explored for the prophylaxis and treatment of COVID-19 daily. In this study, ivermectin was the most commonly used medication despite WHO's advice that it should only be used to treat COVID-19 in clinical trials [28]. This could potentially expose the HCPs to adverse drug reactions, dangerous drug-drug interactions and drug resistance [4].
In addition, commonly used drugs for self-medications during COVID-19 such as hydroxychloroquine and ivermectin became scarce and unaffordable for those who genuinely required the medications for their existing medical conditions such as patients with systemic lupus erythematosus and rheumatoid arthritis thereby putting their health at risk [29, 30].

Occupation was identified as a significant factor associated with self-medication in this study. A higher proportion of pharmacists self-medicated compared to other HCPs which is similar to the report from a previous study by Galvan et al. [31]. This is not surprising because the pharmacists are directly in charge of medicines in the hospital and may 
have easier access to these medications compared to other HCPs. In addition, pharmacists have better knowledge about medicines due to their professional training compared to other HCPs and are likely to practice self-medication more confidently. However, this finding should be interpreted with caution because pharmacists constituted a small proportion of our respondents.

Self-medication was more common in older age group than the younger age group. HCPs older than 44 years were 2.7 times more likely to practice self-medication than those below 44 years. This finding differs from some previous reports that showed that young people were more likely to use self-medication $[1,14]$. The older age group is more vulnerable to COVID-19 with subsequent adverse outcomes than the younger age group [32-34]. In addition, the older HCPs were more likely to have co-morbidities such as hypertension and diabetes mellitus which are contributors to adverse outcomes in COVID-19 [34-36]. This is corroborated by our study that showed that hypertensive and diabetic HCPs were more likely to practice self-medication compared to those without these co-morbidities.

There was a significant association between marital status and practice of self-medication in our study. The HCPs who were single were less likely to practice self-medication. This may be because they were less likely to bother about the possibility of spreading the virus to their spouses and children as would have been expected in married HCPs. Self-medication was also found to be more common in high income earners. This may be because those with high income were more likely to afford the cost of drugs used for self-medication than low income earners.

Those who had COVID-19 screening were 2.7 times more likely to practice self-medication compared to those who had not been screened in this study. Anxiety associated with waiting time for the outcome of the COVID-19 screening result may possibly influence self-medication in this group of HCPs [37].

There was no significant association between educational attainment and practice of self-medication in our study. This is different from findings reported from some previous studies $[5,9,22,31]$ which showed that educated individuals were more likely to practice self-medication due to better access to information and knowledge about drugs [5, 9, 22, 31]. However, Wegbom et al. [10] reported that the less educated individuals were more likely to practice self-medication because of little or no understanding of the adverse implications of self-medication. The absence of significant relationship between educational level and self-medication in our study may be explained by the fact that almost all the HCPs who participated in this study had tertiary level of education.

\section{Limitation}

The report of self-medication among the HCPs involved recall which may introduce bias in determining the prevalence of self-medication. In addition, some HCPs may be unwilling to give information about their self medication practices.

\section{Conclusion}

About one-third of HCPs in our study practiced COVID-19 related self-medication during the pandemic. HCPs that are often assumed to be health literate may not necessarily practice safe health behavior; therefore there is a need for regular health education of the general public including HCPs on the adverse implications of self-medication.

Supplementary Information The online version contains supplementary material available at https://doi.org/10.1007/s11096-021-01374-4.

Acknowledgements The authors acknowledge the efforts of all the research assistants that helped in data collection for this study.

Funding The study was funded by the authors.

Conflicts of interest The authors have no conflict of interest to declare.

\section{References}

1. Onchonga D, Omwoyo J, Nyamamba D. Assessing the prevalence of self-medication among healthcare workers before and during the 2019 SARS-CoV-2 (COVID-19) pandemic in Kenya. Saudi Pharma J. 2020;28:1149-54.

2. Ogboghodo EO, Osaigbovo II, Obarisiagbon OO, et al. FacilityBased Surveillance Activities for COVID-19 Infection and Outcomes among Healthcare Workers in a Nigerian Tertiary Hospital. Am J Tropi Med Hyg. 2021;104:1034-40.

3. World Health Organization Guidelines for regulatory assessment of medicinal products for use inself medication: General Information. WHO Drug Inf 2000;14:18-26.

4. Hughes CM, McElnay JC, Fleming GF. Benefits and risks of self medication. Drug Saf. 2001;24:1027-37.

5. Bamgboye EA, Amoran OE, Yusuf OB. Self medication practices among workers in a tertiary hospital in Nigeria. Afr J Med Med Sci. 2006;35:411-5.

6. Omolase CO, Adeleke OE, Afolabi AO, et al. Self medication amongst general outpatients in a Nigerian community hospital. Ann Ib Postgrad Med. 2007;5:64-7.

7. Bamidele JO, Adebimpe WO, Oladele EA. Knowledge, attitude and use of alternative medical therapy amongst urban residents of Osun State, southwestern Nigeria. Afr J Tradit Complement Altern Med. 2009;6:281-8.

8. Osemene KP, Lamikanra A. A study of the prevalence of selfmedication practice among university students in Southwestern Nigeria. Trop J Pharm Res. 2012;11:683-9.

9. Babatunde OA, Fadare JO, Ojo OJ, et al. Self-medication among health workers in a tertiary institution in South-West Nigeria. Pan 
Afr Med J. 2016. https://doi.org/10.11604/pamj.2016.24.312. 8146.

10. Wegbom AI, Edet CK, Raimi O, et al. Self-Medication Practices and Associated Factors in the Prevention and/or Treatment of COVID-19 Virus: A Population-Based Survey in Nigeria. Front Public Health. 2021;9:606801.

11. Obuaku C. Essential medicines in Nigeria: foregrounding access to affordable essential medicines. Afr Sociol Rev/Rev Afr Sociol. 2014;18:42-60.

12. Onwujekwe O, Onoka C, Uzochukwu B, Hanson K. Constraints to universal coverage: inequities in health service use and expenditures for different health conditions and providers. Int $\mathrm{J}$ Equity Health. 2011;10:1-9.

13. Garuba HA, Kohler JC, Huisman AM. Transparency in Nigeria's public pharmaceutical sector: perceptions from policy makers. Global Health. 2009;5:1-13.

14. Tobin EA, Erhazele J, Okonofua M, et al. Self-medication among health care workers in a tertiary hospital in Southern Nigeria: knowledge, attitude, and practices. Med J Indones. 2020;29:403-9.

15. Bandyopadhyay $S$, Baticulon RE. Kadhum $M$ et al Infection and mortality of healthcare workers worldwide from COVID-19: a systematic review. BMJ Glob Health. 2020;5:e003097.

16. Onchonga D, Ngetich E, Makunda W, et al. Anxiety and depression due to 2019 SARS-CoV-2 among frontier healthcare workers in Kenya. Heliyon. 2021;7:e06351.

17. Costanzo M, De Giglio MAR, Roviello GN. SARS-CoV-2: recent reports on antiviral therapies based on lopinavir/ritonavir, darunavir/umifenovir, hydroxychloroquine, remdesivir, favipiravir and other drugs for the treatment of the new coronavirus. Curr Med Chem. 2020;27:4536-41.

18. Principi N, Esposito S. Chloroquine or hydroxychloroquine for prophylaxis of COVID-19. Lancet Infect Dis. 2020;20:1118.

19. Nigerian Centre for Disease Control. National interim guidelines for clinical management of COVID-19, version 3, June 2020, Abuja. Nigeria Accessed. 2010;24:10.

20. Quispe-Cañari JF, Fidel-Rosales E, Manrique D, et al. Selfmedication practices during the COVID-19 pandemic among the adult population in Peru: A cross-sectional survey. Saudi Pharm J. 2021;29:1-11.

21. Osaigbovo II, Ogboghodo EO, Obaseki D, et al. Pattern of drug sales at community pharmacies in edo state as evidence of selfmedication during the COVID-19 pandemic: implications for policy implementation. Niger Health J. 2021;20:150-8.

22. Sadio AJ, Gbeasor-Komlanvi FA, Konu RY, et al. Assessment of self-medication practices in the context of the COVID-19 outbreak in Togo. BMC Public Health. 2021;21(1):1-9.
23. Ahmad AR, Murad HR. The impact of social media on panic during the COVID-19 pandemic in Iraqi Kurdistan: online questionnaire study. J Med Int Res. 2020;22:e19556.

24. Sabetian G, Moghadami M, Haghighi LH, et al. COVID-19 infection among healthcare workers: a cross-sectional study in southwest Iran. VirolJ. 2021;18:1-8.

25. Zeballos Rivas DR, Lopez Jaldin ML, Nina Canaviri B, et al. Social media exposure, risk perception, preventive behaviors and attitudes during the COVID-19 epidemic in La Paz, Bolivia: A cross sectional study. PLoS ONE. 2021;16:e0245859.

26. Makowska M, Boguszewski R, Nowakowski M, et al. Self-medication-related behaviors and Poland's COVID-19 lockdown. Int J Environ Res Public Health. 2020;17(22):8344.

27. New York Times. Coronavirus has become a pandemic, WHO says 11th March 2020. Accessed. 2021;20:08.

28. WHO advises that ivermectin only be used to treat COVID-19 within clinical trials. Available from:https://www.who.int/newsroom/feature-stories/detail/who-advises-that-ivermectin-only-beused-to-treat-covid-19-within-clinical-trials. Accessed 13:08:2021

29. Peschken CA. Possible consequences of a shortage of hydroxychloroquine for patients with systemic lupus erythematosus amid the COVID-19 pandemic. J Rheumatol. 2020;47:787-90.

30. Mendel A, Bernatsky S, Thorne JC, et al. Hydroxychloroquine shortages during the COVID-19 pandemic. Ann Rheum Dis. 2021;80:e31.

31. Galvan MR, Dal Pai D, Echevarría-Guanilo ME. Self medication among health professionals. Rev Min Enferm. 2016;20:e959.

32. Hu C, Li J, Xing X, et al. The effect of age on the clinical and immune characteristics of critically ill patients with COVID-19: A preliminary report. PLoS ONE. 2021;16(3):e0248675.

33. Aliberti MJR, Covinsky KE, Garcez FB, et al. A fuller picture of COVID-19 prognosis: the added value of vulnerability measures to predict mortality in hospitalised older adults. Age Ageing. 2021;50:32-9.

34. Li X, Xu S, Yu M, et al. Risk factors for severity and mortality in adult COVID-19 in patients in Wuhan. J Allergy Clin Immunol. 2020;146:110-1.

35. Albitar O, Ballouze R, Ooi JP, et al. Risk factors for mortality among COVID-19 patients. Diabetes Res Clin Pract. 2020;166:108293.

36. Huang S, Wang J, Liu F, et al. COVID-19 patients with hypertension have more severe disease: a multicenter retrospective observational study. Hypertens Res. 2020;43:824-31.

37. Sahu DP, Pradhan SK, Sahoo DP, et al. Fear and anxiety among COVID-19 screening clinic beneficiaries of a tertiary care hospital of Eastern India. Asian J Psychiatr. 2021;57:102543.

Publisher's Note Springer Nature remains neutral with regard to jurisdictional claims in published maps and institutional affiliations. 